\title{
Recovery of the electron-phonon interaction function in superconducting tantalum ballistic contacts
}

\author{
N. L. Bobrov ${ }^{1}$ \\ ${ }^{1}$ B. Verkin Institute for Low Temperature Physics and Engineering, 47, Nauki Ave., 310164 Kharkov, Ukraine \\ Email address: bobrov@ilt.kharkov.ua
}

(Dated: March 9, 2021; Published Fiz. Nizk. Temp., 45, 562 (2019); [Low Temp. Phys. 45, 482(2019)])

The experimentally observed nonlinearities of the current-voltage characteristics (CVCs) of tantalum-based point homo- and hetero- contacts in both normal and superconducting states related to electron-phonon interaction (EPI) were analyzed. It was taken into account that additional nonlinearity of CVCs arising upon contact transition to the superconducting state (superconducting spectral component) is formed not only near the constriction in the region roughly equal to the contact diameter (as is the case for the normal state, and as predicted theoretically for the superconducting state), but also in a markedly larger region that is about the size of the coherence length. In this case, a considerable role in the formation of this superconducting component is played by nonequilibrium phonons with low group velocity, which account for the experimentally observed sharpening of the phonon peaks in the EPI spectra (the second derivatives of the CVCs) during the superconducting transition of the contacts, instead of the theoretically expected peak broadening (spreading), and for the increase in the superconducting contribution to the point contact spectrum in the low and medium energy regions. The high-energy part of the EPI spectrum changes much less significantly during the superconducting transition, which is attributable to suppression of the excess contact current by nonequilibrium quasi-particles. A detailed procedure was proposed for the recovery of the EPI spectral function from the point contact spectrum contribution (the second derivative of the CVC) that arises during the superconducting transition of one or both contacting metals.

PACS numbers: 71.38.-k; 73.40.Jn; 74.25.Kc; 74.45.+c

\section{INTRODUCTION}

The usage of the nonlinearity of the current-voltage characteristics (CVC) of ballistic point contacts in the normal state to restore the electron-phonon interaction (EPI) function in metals is well known. Several hundred publications are available on this subject and two integrating monographs have been published [1,2]. Electron duplication takes place for these contacts in the on-state, namely, the electrons are split into two groups for which the energy difference between the occupied and unoccupied states on the Fermi surface is $e V$ [3]. In other words, an electron that has traveled through the constriction from the opposite contact bank differs in energy from electrons within the present bank by exactly the applied voltage value. At any point of the trajectory, the electron may lose excess energy by emitting a nonequilibrium phonon with the energy $\mathrm{eV}$. Since the contact is ballistic, the average energy relaxation length is much greater than the contact size.

Thus, the greater part of nonequilibrium phonon emissions would occur away from the constriction, within the contact banks. If after scattering, the electron goes back through the constriction to the initial bank of the point contact from which it flew out with an additional energy, the contact resistance would increase. These processes are referred to as backscattering. These processes provide the basis for the Yanson EPI spectroscopy where the second derivative of the contact CVC directly reflects the structure of the EPI function. It is obvious that, since during excess energy electron scattering via emission of nonequilibrium phonons, in the isotropic case, the direction of electron movement can change in an arbitrary way, then, due to geometric considerations, the backscattering processes are efficient only in a volume relatively proximate to the constriction. If the contact is shaped like an orifice of diameter $d$ in a thin wall, this volume is approximately equal to the volume of a sphere with the same diameter [4]. Hence, most of the electron scattering on nonequilibrium phonons does not participate in the formation of the EPI spectrum in Yanson spectroscopy.

The transition to the superconducting state brings about an additional CVC nonlinearity caused by the suppression (decrease) of the excess current (CVC difference 
between the superconducting and normal states of the contact metal at the same voltage) upon reabsorption of nonequilibrium phonons by Andreev-reflected electrons (hereinafter referred to as Andreev electrons, for brevity). This nonlinearity can also be used for the recovery of the EPI function. The appropriate theories that allow this to be done for S-c-N and S-c-S contacts ( $\mathrm{S}$ is superconductor, $\mathrm{N}$ is normal metal, $\mathrm{c}$ is constriction) appeared in $1983[5,6]$; however, practical attempts to recover the EPI function from the superconducting characteristics of these contacts were made relatively recently [7-9].

For tantalum-based contacts, the previously used procedure for EPI function recovery required further development, taking account of the ratio between the contact size and the coherence length. The approaches considered in this article may prove to be fairly important in the analysis of experimental data of point contact EPI spectroscopy in superconductors.

\section{THEORETICAL GROUNDS}

Reabsorption processes, in other words, Andreev electron reflections on nonequilibrium phonons, are not subject to geometrical constraints typical of backscattering processes; any reflection process is effective. These processes can take place in the volume where nonequilibrium phonons and Andreev electrons coexist simultaneously. Since the conversion of Andreev electrons to Cooper pairs occurs at the reduced coherence length $\zeta$ $\left(\frac{1}{\zeta}=\frac{1}{\xi_{0}}+\frac{1}{l_{i}}, \xi_{0}\right.$ is the superconducting coherence length, $l_{i}$ is the length of scattering on impurities), then the volume, in the isotropic case, is equal to the volume of a sphere (or hemisphere for S-c-N point contacts) with the radius $\zeta$.

However, for ballistic contacts with great coherence and elastic relaxation lengths appearing in the theory, these processes are fairly probable in approximately the same volume as backscattering. Here the volume restriction is caused by fast decrease in the concentrations of both nonequilibrium phonons and Andreev electrons moving away from the constriction. High current density in the vicinity of the orifice provides also their high concentration, which rapidly declines as the current spreads. Therefore, at large distances $r$ from the constriction, the contact can be considered to be a point source of phonons, with their density decreasing as $\sim 1 / r^{2}$. Since the minimum size in which the superconducting energy gap can vary coincides with the length $\zeta \gg d(d$ is the contact diameter), these scattering processes do not change the gap in the near-contact region, and suppression of the excess current is due to a minor decrease in the quantity of Andreev electrons.

The Khlus and Omel'yanchuk theory [5, 6] of inelastic point contact EPI spectroscopy in the S-c-S and S-c-N point contacts was developed for ballistic contacts, i.e., for those contacts that obey the condition $d \ll \zeta, v_{F} / \omega_{D}$ ( $v_{F}$ is the Fermi velocity, $v_{F} / \omega_{D} \sim l_{\varepsilon}$, where $l_{\varepsilon}$ is the energy free path at the Debye energy $\left.\hbar \omega_{D}\right)$.

For S-c-S contacts, it was found that [5]

$$
\frac{d I_{\text {exc }}}{d V}(V)=-\frac{64}{3 R}\left(\frac{\Delta L}{\hbar \bar{v}}\right)\left[G^{N}(\omega)+\frac{1}{4} G^{S}(\omega)\right]_{\omega=e V / \hbar}
$$

Here $R$ is the contact resistance, $L$ is a function that is fairly sophisticated for arbitrary arguments, $\bar{v}$ is the velocity of electrons averaged over the Fermi surface, $G^{N}(\omega)$ is the point contact (PC) EPI function, which is the same as that of point contacts in the normal state according to the Kulik-Omel'yanchuk-Shekhter (KOS) theory [2], $G^{S}(\omega)$ is the superconducting PC EPI function differing from $G^{N}(\omega)$ by a form factor, $\Delta$ is the superconducting energy gap. Furthermore, unlike the normal form factor, which is responsible for the backscattering contribution to the current, in the case of the superconducting form factor appearing in $G^{S}(\omega)$, the contribution to the current is made by electron-phonon collisions associated with Andreev reflection-like processes in the region of the contact, i.e., by of the conversion of quasi-electron excitations to quasi-holes. A similar expression was obtained for the S-c-N contact [6]:

$$
\begin{gathered}
\frac{1}{R(V)}-\left(\frac{1}{R(V)}\right)_{\Delta=0}= \\
=-\frac{32}{3 R} \times \frac{d \Delta}{h} \times\left[\frac{1}{v_{F}^{(1)}} \times G_{1}(\omega)+\frac{1}{v_{F}^{(2)}} \times G_{2}(\omega)\right]
\end{gathered}
$$

Here $G_{i}(\omega)(i=1,2)$ is the EPI function of metals that form the point contact.

The relative phonon contribution to the excess current at $e V \sim \omega_{D}$ is about $d \cdot \omega_{D} / v_{F}$, i.e., it is small when the condition $d \ll v_{F} / \omega_{D}$ holds. This small contribution is very important to ensuring that equal changes in the nonequilibrium phonon flux density for different biases at the contact cause equal changes in the excess current. Meanwhile, when suppression of the excess current is pronounced, this relationship is violated. For this type of contacts, recovery of the EPI function would require a correction of the useful signal amplitude in the region of decreasing excess current [9]. 
For ballistic contacts, the current spreading results in Andreev electrons being spread over a larger space in which the concentration of nonequilibrium phonons is low; therefore, the relative decline of excess current for these contacts is minor. The nonlinear deviation of the point contact resistance from $R_{0}=R$ at $V=0$ caused by scattering of nonequilibrium phonons on Andreev electrons is several times smaller than that caused by backscattering processes. As a result, if $\mathrm{eV} \gg \Delta$, the point contact spectrum changes insignificantly upon transition to the superconducting state. Khlus [6] described this transformation of the second derivative of the CVC for the ballistic S-c-N point contact:

$$
\frac{1}{R} \cdot \frac{d R}{d V}=\frac{16 e d}{3 \pi} \cdot \sum_{i=1,2} \frac{1}{v_{F}^{(i)}} \cdot \int_{0}^{\infty} \frac{d \omega}{\Delta} \cdot S\left(\frac{\omega-e V}{\Delta}\right) G_{i}(\omega) .
$$

where $G_{i}(\omega)$ are the EPI functions of the normal and superconducting metals that form the heterocontact, $S(x)$ is the smearing factor:

$$
S(x)=\theta(x-1) \frac{2\left(x-\sqrt{x^{2}-1}\right)^{2}}{\sqrt{x^{2}-1}},
$$

here $\theta(y)$ is the Heaviside theta function.

Upon the superconducting transition, the spectrum is additionally smeared and at $T \rightarrow 0$, the resolution is determined by the value of $\Delta$. In view of the relationship between the CVC derivative and the PC EPI function, it follows from expression (3) that

$$
\tilde{g}_{p c}^{S}(e V)=\int_{0}^{\infty} \frac{d \omega}{\Delta} S\left(\frac{\omega-e V}{\Delta}\right) g_{p c}^{N}(\omega)
$$

Thus, with the point contact spectra of the heterocontact in the normal and superconducting states at hand, one can compare the results of calculations and experiments. In addition, as shown by Bobrov et al. [8],

$$
g_{p c}^{S}(e V)=\frac{1}{\Delta} \int_{0}^{e V}\left[\tilde{g}_{p c}^{S}(\omega)-g_{p c}^{N}(\omega)\right] d \omega
$$

Equation (6) obviously follows from the fact that the first derivative of the excess current is proportional to the EPI current. It can be seen that the function $\tilde{g}_{p c}^{S}(\mathrm{eV})$ reflects the transformation of the spectrum as the contact goes to the superconducting state. It is proportional to the CVC second derivative, shifted to lower energy by a value of about $\Delta$, additionally broadened and, as a result, it has a slightly lower intensity. The function $g_{p c}^{S}(e V)$

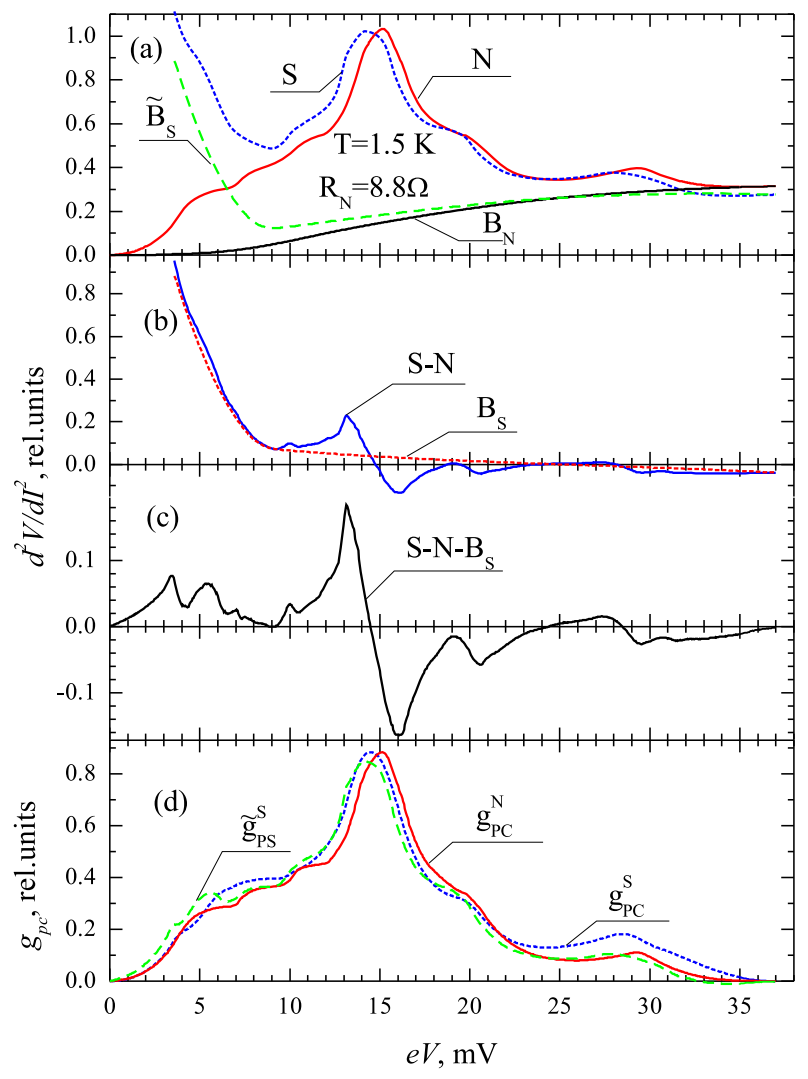

Figure 1. (a) EPI spectra of the Sn-Cu point contact in the normal and superconducting states. $\tilde{B}_{S}$ and $B_{N}$ are the background curves for the superconducting and normal spectra, respectively. The superconductivity is suppressed by a magnetic field. (b) Difference between the superconducting and normal spectra and the assumed shape of the background curve. (c) The difference curve (after background subtraction). (d) Point contact EPI functions recovered from the normal and superconducting states and by integration of the difference curve. For the convenience of comparison, the curve $g_{p c}^{S}$ is aligned in amplitude with the curve $g_{p c}^{N}$.

is proportional to the first derivative of excess current and does not contain additional broadening; according to calculations, its shift to lower energy is approximately two times less than that of $\tilde{g}_{p c}^{S}(e V)$.

\section{COMPARISON OF THE THEORETICAL CONCLUSIONS WITH THE EXPERIMENT. TIN-BASED POINT CONTACTS}

As an example, first, we will consider tin-based point contacts, which show good agreement between the theory and the experiment [8]. Figure 1 presents the spectra of a $\mathrm{Sn}-\mathrm{Cu}$ point contact in the normal and superconducting 
states. The difference curve and the background curves, $B_{N}, B_{S}$, and $\tilde{B}_{S}$, are also given. It turned out that background is inherent in not only the Yanson point contact spectroscopy. The background $\mathrm{BN}$ is clearly manifested as a nonzero second derivative of the CVC at displacements greater than those corresponding to the boundary frequency of the phonon spectrum, whereas the EPI function goes to zero. The background is determined most often by the self-consistent iteration procedure [1].

It was found that mere integration of the difference curve $\left(d^{2} V / d I^{2}\right)_{S}-\left(d^{2} V / d I^{2}\right)_{N}$ is insufficient for obtaining the curve proportional to the EPI function $g_{p c}^{S}(e V)$, since apart from the effect related to the gap at low biases, the second derivatives also differ in the background level beyond the boundary of the phonon spectrum. This brings about the necessity to subtract the background curve $B_{S}$ from the difference curve before integration. Apart from the obvious condition of being equal to zero at energies exceeding the maximum phonon spectrum frequency, the curve obtained after background subtraction should obey the rule of sums: the total areas under the curves above and below the abscissa axis must be equal.

The rule of sums follows from the fact that integration of the obtained curve gives a curve with a zero background. It is clear that these criteria can be met for a multitude of different background curves; however, their variations, provided they are monotonic, do not induce considerable changes in the shapes and positions of phonon effects of the EPI function being recovered.

In order to verify the theory predictions, it is necessary to follow the transformation of the spectrum upon transition from the normal to superconducting state without the background component $\tilde{B}_{S}$, in order to get a curve proportional to $\tilde{g}_{p c}^{S}(\omega)$. In the absence of a normal state spectrum and difference curve with subtracted backgrounds, this is difficult to perform; however, when these curves are available, this problem is easily solved: let $\tilde{S}$ be the spectrum in the superconducting state with background subtracted. Then $\tilde{B}_{S}=S-\tilde{S}=S-\left[\left(N-B_{N}\right)+\left(S-N-B_{S}\right)\right]=B_{N}+B_{S}$. The latter is even somewhat more convenient, as this allows for an easier approximation of the missing part of the curve in the small bias region near the gap effect. For the convenience of comparison, the curve $g_{p c}^{S}$ in Fig.1(d) is aligned in amplitude with the curve $g_{p c}^{N}$. As can be seen from comparison of Figs.1(a) and 1(d), the theory predictions are in good agreement with the experiment.

Upon transition to the superconducting state, the $\tilde{g}_{p c}^{S}(\omega)$ curves smear, decrease in the amplitude, and shift to lower energies by a value approximately equal to the gap. The shapes of experimental and theoretical $g_{p c}^{S}$ curves differ in the high-energy region: the experimental curve is markedly more intense. Apparently, this is attributable to increasing concentration of nonequilibrium phonons at the contact periphery caused by the decrease in the energy relaxation length of electrons close to the Debye energy.

\section{COMPARISON OF THE CHARACTERISTIC PARAMETERS FOR TIN AND TANTALUM}

It turns out that even the complete correspondence of the point contact parameters to the requirements of the theory does not ensure that the experimental curves follow the theoretical model. For example, as it was ascertained for ballistic point contacts based on tantalum [10-12], the superconducting transition is accompanied by a radical change in the shape, intensity, and positions of phonon effects. Furthermore, differences from theory predictions are observed for both hetero- and homocontacts. Instead of the expected broadening of the phonon peaks, they become strongly sharpened. As a consequence, the amplitude of these peaks substantially increases.

In addition, the soft phonon mode, which is manifested in the normal state as a shoulder, is converted to a sharp peak as the contacts switch to the superconducting state. This is manifested most vividly for relatively low-resistance point contacts, but even for highresistance contacts, in which the ballistic condition is satisfied rather strictly, the deviations from theory predictions are quite large. In order to understand what could be responsible for these crucial differences in the behaviors of tantalum- and tin-based point contacts, compare their characteristic parameters summarized in Table I.

Since the Table presents the energy relaxation length at the Debye energies, $l_{\varepsilon}^{D}$, and the deviations in the tantalum spectra from the theory predictions already start at small biases, it appears reasonable to compare these lengths over the whole range of biases. The inelastic mean free path of electrons for an arbitrary contact voltage can be estimated from the formula $\frac{1}{l_{\varepsilon}(e V)}=\frac{2 \pi}{\hbar v_{F}} \int_{0}^{e V} d \omega g(\omega)$, where $g(\omega)$ is the EPI function. Figure 2 shows the point contact EPI functions for Ta [13] and Sn [1] that were used to estimate the energy 
Table I. Characteristic parameters of tantalum- and tin-based point contacts.

\begin{tabular}{|c||c|c|c|c|c|c|c|c|c|c|}
\hline & $\mathrm{R}, \mathrm{Ohm}$ & $\mathrm{d}, \mathrm{nm}$ & $\rho_{300} / \rho_{\text {res }}$ & $\rho l, \mathrm{Ohm} \cdot \mathrm{cm}^{2}$ & $v_{F}, \mathrm{~cm} / \mathrm{s}$ & $l_{\varepsilon}^{D}, \mathrm{~nm}$ & $l_{i}, \mathrm{~nm}$ & $\xi_{0}, \mathrm{~nm}$ & $\zeta, \mathrm{nm}$ & $\Delta, \mathrm{mV}$ \\
\hline \hline $\mathrm{Sn}$ & $7 \div 30$ & $5.1 \div 10.5$ & $\sim 15000$ & $4.5 \cdot 10^{-12}$ & $1.89 \cdot 10^{8}$ & $\sim 360$ & 6000 & $\sim 200$ & $\sim 200$ & 0.57 \\
\hline $\mathrm{Ta}$ & $15 \div 210$ & $2.2 \div 8.5$ & 18 & $5.9 \cdot 10^{-12}$ & $0.74 \cdot 10^{8}$ & $\sim 90$ & 82 & 92 & 43 & 0.71 \\
\hline
\end{tabular}

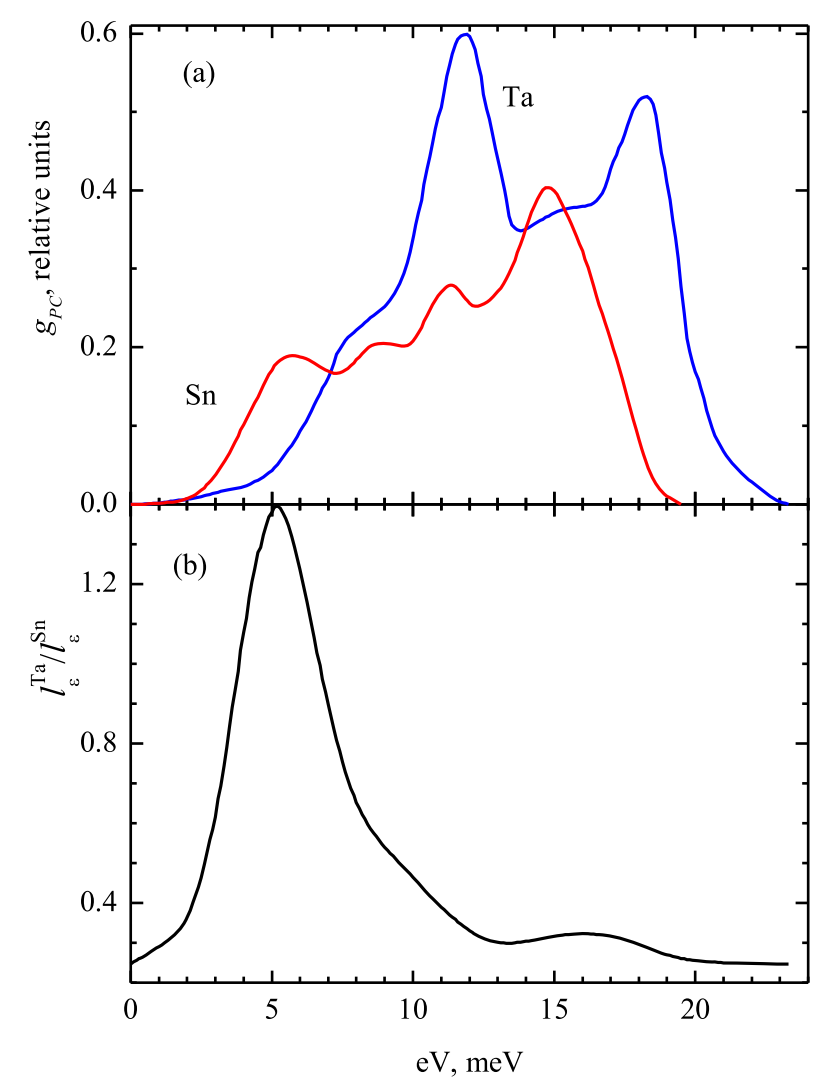

Figure 2. (a) Point contact EPI functions for tin and tantalum used to estimate the energy relaxation rate vs. the contact bias. (b) Energy relaxation lengths for tantalum in relation to tin vs. the bias.

relaxation lengths. The estimates were made using the electron parameters presented in the Table. As can be seen in Fig.2, the relaxation length is greater in tantalum than in tin and is in the $4-7 \mathrm{meV}$ range, which is close to the soft mode energy in tantalum. At other energies, $l_{\varepsilon}$ is smaller in tantalum than in tin; it is approximately four times smaller at the Debye energies.

Considering these estimates, one can conclude that the reduced coherence length $\zeta$ is the key factor responsible for the deviation from the theory's predictions. As follows from the Table, this value for tin coincides with the coherence length, $\zeta \approx \xi_{0}$, in view of the very large elastic scattering length $l_{i}$, while in tantalum, $\zeta \sim 43 \mathrm{~nm}$, i.e., the volume of the sphere limited by the reduced coher- ence length is 100 times smaller in tantalum than in tin. For these reasons, and because of the lower Fermi velocity of electrons in tantalum, the concentration of Andreev electrons in this volume increases compared with that for tin. Therefore, reabsorption of nonequilibrium phonons by Andreev electrons starts to play a noticeable role not only in the volume roughly equal to the contact diameter, as in tin, but also in the volume with the characteristic size of the reduced coherence length. In other words, the excess current decreases not only because of decreasing quantity of the Andreev electrons within a volume about equal to the contact diameter, but also because of suppression of the gap in the contact region with a volume about equal to the coherence length.

\section{NONEQUILIBRIUM EFFECTS IN TANTALUM-BASED POINT CONTACTS}

Before considering specific features of the formation mechanism of phonon peaks in the tantalum-based superconducting contacts, we will pay attention to other nonlinear effects manifested in the CVCs of these contacts, which are also associated with the small coherence length. These effects are not spectral and their position in the energy axis depends on both the point contact resistance and the temperature and magnetic field strength. In the second derivative of the CVC, the lowtemperature effect occurs immediately after the gap effect as a narrow and sharp surge and is greater in intensity. This corresponds to a stepwise decrease in the excess current caused by the abrupt decrease in the superconducting gap in the region adjoining the contact. This phenomenon is associated with the attained critical density of nonequilibrium quasi-particles in the near-contact region and is considered in detail in Refs.[10-12]. Since the contacts investigated here are ballistic, most of electrons lose excess energy within the point contact banks, when scattered on nonequilibrium phonons.

In the superconducting state these electrons that have lost the excess energy are accumulated above the gap. With increasing contact bias, the quantity of these nonequilibrium quasi-particles increases; when some crit- 
ical concentration is reached, a part of the superconductor adjoining the constriction abruptly switches to a new, nonequilibrium state with a partly suppressed gap. It is obvious that the smaller the space in which this switching takes place, the more rapidly the required concentration is reached. The minimum size of this region cannot be smaller than the reduced coherence length $\zeta$. The position of the nonequilibrium effect at a specified temperature for contacts with different resistance corresponds to the same injection power, being approximately $0.4 \mu \mathrm{W}$ at $2 \mathrm{~K}$.

As the temperature or magnetic field increases, the relaxation rate of these quasi-particles above the gap increases; therefore, for attaining the critical concentration, it is necessary to increase the injection power. This shifts the effect to higher voltages. This rules out the interpretation of these effects as being due to the degradation of superconductivity caused by heating or suppression by the magnetic field. Since the stepwise decrease in the excess current upon transition to the nonequilibrium state is insignificant, the change in the gap accompanied by the transition is also moderate. Thus, the influence of the nonequilibrium effect on the superconducting spectral contribution is also small, except that it can be located at biases corresponding to some phonon effect and thus hamper observation of the phonon effect. However, as follows from experimental results, the suppression of the excess current is not limited to the stepwise section resulting from the phase transition to a new nonequilibrium state.

On further increase of the voltage applied to the contact and, hence, increase in the injection power, the suppression of the excess current is rather smooth and is not accompanied by any indications in the CVC derivatives. Depending on the resistance, this suppression may be rather small for high-resistance contacts and fairly large (several-fold larger) for low-resistance contacts. In any case, this suppression has a pronounced influence on the generation of EPI-related effects.

\section{EFFECT OF THE NEAR-CONTACT REGION ON THE FORMATION OF THE SPECTRUM}

Now we will consider the mechanism of formation of phonon effects related to the excess current. Conventionally speaking, they represent a superposition of contributions from two spatially different regions: a contribution from the region about equal to point contact diameter, which corresponds to the theoretical model, and a contribution of the near-contact region about equal to the coherence length $\zeta$. In principle, the mechanism of formation of phonon effects is the same in both regions and is related to reabsorption of nonequilibrium phonons by Andreev electrons. However, since the second region is much larger than the contact diameter, here a considerable role is played by differences between the group velocities of nonequilibrium phonons generated by electrons with the excess energy $\mathrm{eV}$.

Since phonons with energies corresponding to the maximum density of states have the lowest group velocities, $\partial \omega / \partial q=0$, these phonons would leave this region more slowly and would thus be accumulated in a higher concentration. Since the relative concentrations of nonequilibrium phonons and Andreev electrons determine the value of the negative contribution to the excess current, the greatest contribution to the spectrum will occur at the maximum density of phonon states. This assumption is supported by sharpening of the phonon peaks. In the region approximately equal to the contact diameter, because of small volume, slow phonons do not have time to concentrate, and their specific contribution to the nonlinearity would be the same as that of phonons with higher group velocity. In terms of this model, it is clear that for selection of phonons with low group velocities, fast phonons must be free to leave the near-contact volume, i.e., the flight of phonons should correspond to the ballistic regime.

Thus, the ratio of contributions of the near-contact and remote regions would influence the degree of sharpening of phonon effects. Evidently, the increase in the contact size would lead to increasing proportion of the latter contribution and, hence, to increasing degree of sharpening of phonon effects. As shown below, this assumption is perfectly confirmed by experimental results.

\section{TA-CU HETEROCONTACTS}

Unlike the spectra of Sn-Cu heterocontacts, the spectra of $\mathrm{Ta}-\mathrm{Cu}$ heterocontacts do not show the contribution of copper [13]. In the spectra of heterocontacts of transition $d$-metals with $\mathrm{Cu}, \mathrm{Ag}$, and $\mathrm{Au}$, the contribution of the latter is not manifested. To begin with, consider a high-resistance (209 ohm) Ta-Cu heterocontact (see Fig.3). Formally, it satisfies all requirements of the theory: its diameter is $\approx 2.2 \mathrm{~nm}$, whereas the elastic 


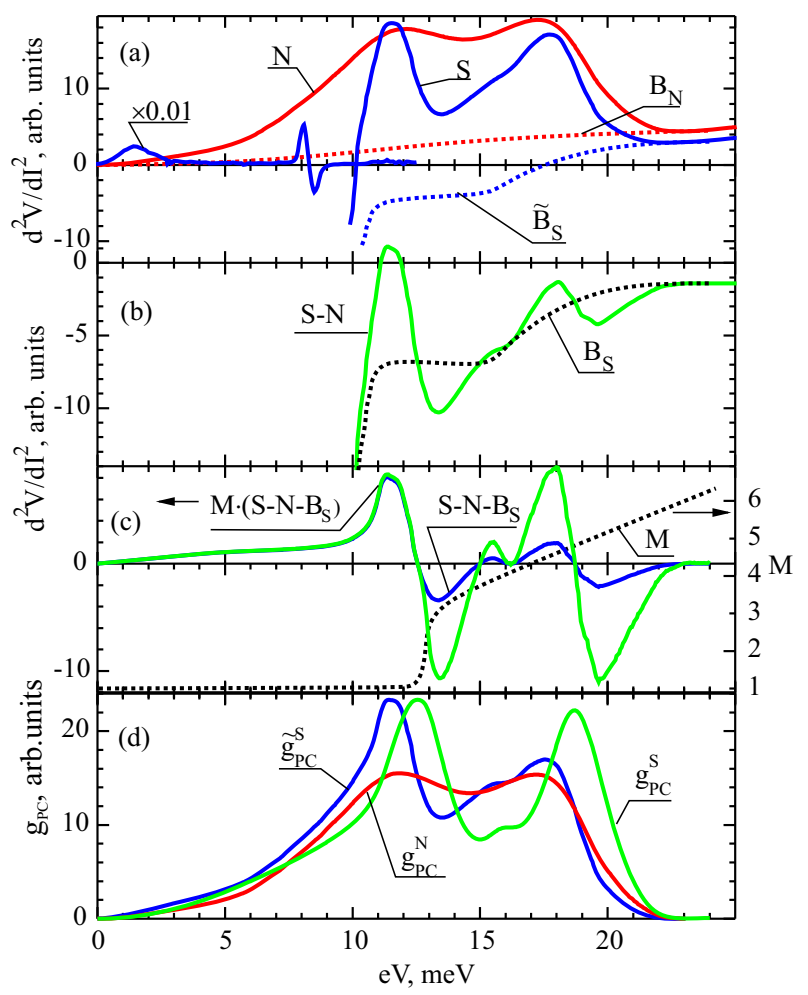

Figure 3. (a) EPI spectra of the $\mathrm{Ta}-\mathrm{Cu}$ point contact in the normal and superconducting states. $T=1.4 K, R_{0}=210 \mathrm{ohm}$; the initial segment of the superconducting curve containing the gap and nonequilibrium effects is scaled down by a factor of $100, \tilde{B}_{S}$ and $B_{N}$ are the background curves for the superconducting and normal spectra, respectively. (b) The difference between the superconducting and normal spectra, and the assumed shape of the background curve. (c) Difference curve (after background subtraction), scaling curve M, and the difference curve multiplied by the scaling curve. (d) Point contact EPI functions recovered from the spectra for normal and superconducting states and from the superconducting contribution to the spectrum by integration of the corrected difference curve (c). For convenience of comparison, the curve $g_{p c}^{S}$ is aligned in amplitude with the curve $\tilde{g}_{p c}^{S}$. The scale is the same in all panels of the Figure.

scattering length in the tantalum bank of the contact at the liquid helium temperature is $\approx 84 \mathrm{~nm}$, the energy relaxation length at the Debye energy is approximately the same $(\approx 90 \mathrm{~nm})$, and the reduced coherence length in tantalum is $\zeta \approx 43 \mathrm{~nm}$; in other words, all of the lengths are more than an order of magnitude greater than the contact diameter. Nevertheless, the transformation of the spectrum upon transition to the superconducting state differs crucially from predictions of the theory for ballistic S-c-N point contacts.

Pay attention to the region of phonon energies. First, in the superconducting state, there is no spectral smearing and no related decrease in the intensity. Conversely, the phonon modes are markedly sharpened and their amplitude increases. Since the resistance of this contact is very high, the above-noted nonequilibrium effect is located in a rather high-energy region and coincides with the soft phonon mode; therefore, it is impossible to follow the transformation of this mode upon the superconducting transition. Regarding higher frequency regions of the spectrum, they can be examined most conveniently in Fig.3(d), which shows the curves after background subtraction. A comparison of $g_{p c}^{N}$ and $\tilde{g}_{P C}^{S}$ demonstrates that upon transition to the superconducting state, the amplitude of the first phonon peak increases to the highest extent, while the growth of the high-energy peak is much less pronounced. Therefore, it is impossible to perform a correct recovery of the EPI function from the superconducting contribution to the spectrum, after the same background subtraction procedure as was done for tin, by integration of the $\mathrm{S}-\mathrm{N}-B_{S}$ curve, because the rule of sums is not satisfied in this case. This inconsistency was resolved by making a correction of the shape of the S$\mathrm{N}-B_{S}$ curve, which was done by multiplying it by the scaling curve M [see Fig.3(c)]. This empirical curve does not change the difference curve in the low-frequency region and increases its amplitude in the high-frequency region.

The result of recovery before and after the correction can be observed in Fig.4. The decrease in the amplitude of the superconducting contribution to the spectrum in the high-energy region is caused by suppression of the excess current in the contact by nonequilibrium quasi-particles mentioned above in the discussion dealing with nonspectral nonequilibrium effects in superconducting curves. Figure 5 presents the differential resistances of the point contact in the normal and superconducting states, the dependence of the excess current on the voltage, and the scaling curve for correction of the amplitude of the superconducting contribution to the spectrum [the same as in Fig.3(c)]. The vertical and horizontal segments refer to the voltage and excess current corresponding to the onset of suppression of the amplitude of the superconducting contribution to the spectrum.

Let us consider the influence of the decrease in the point contact resistance. Figure 6 shows the spectra of the point contact with $73 \mathrm{ohm}$ resistance. In this case, the nonequilibrium effect already occurs at about $\sim 5 \mathrm{mV}$ and coincides with the energy of the tantalum soft 


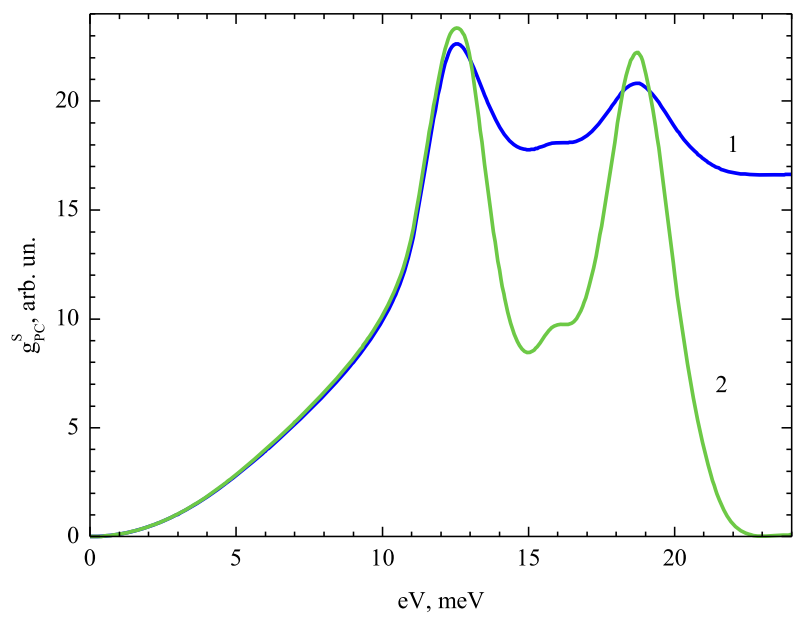

Figure 4. A demonstration of how violating the rule of sums impacts the shape of the recovered EPI function. EPI function with the background (1) recovered without accounting for the correction of the suppressed high-frequency part of the S-N- $B_{S}$ curve [see Fig.3(c)] and without the background (2) recovered from the corrected curve $\mathrm{M}\left(\mathrm{S}-\mathrm{N}-B_{S}\right)$. The scale of the curves is the same as that in Fig.3.

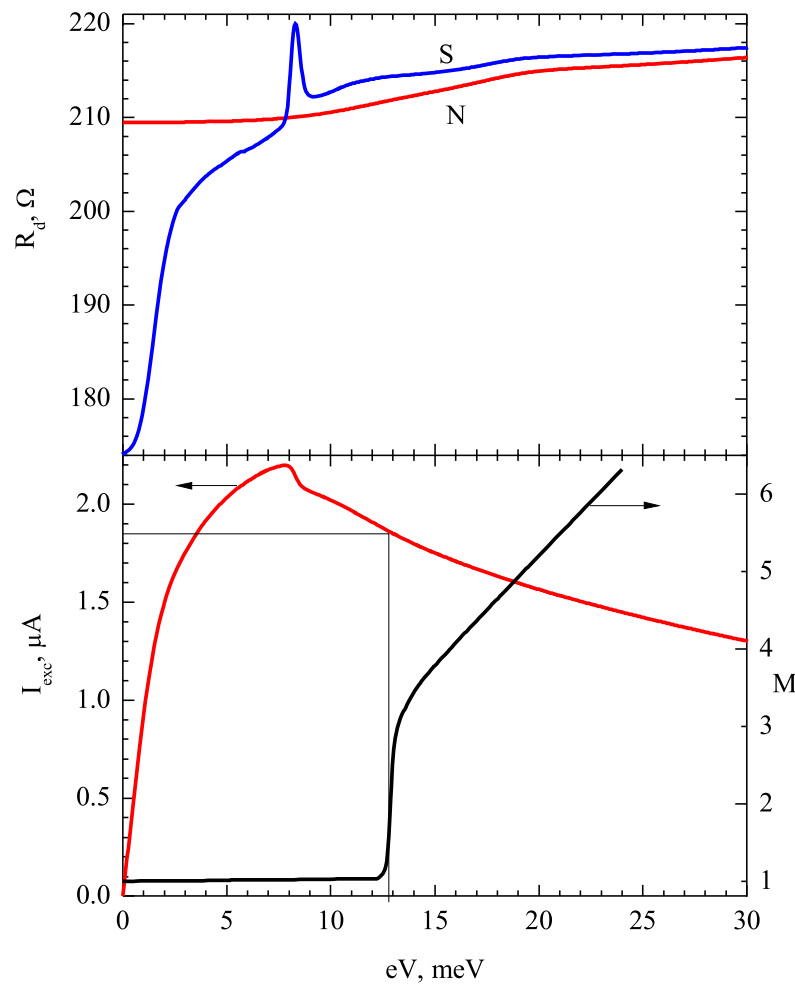

Figure 5. Differential resistance of the point contact whose characteristics are shown in Fig.3 in the normal (N) and superconducting $(\mathrm{S})$ states and excess current vs. the bias. The excess current panel shows the scaling curve M (see Fig.3) with designated excess current and voltage values corresponding to the step position in the scaling curve.

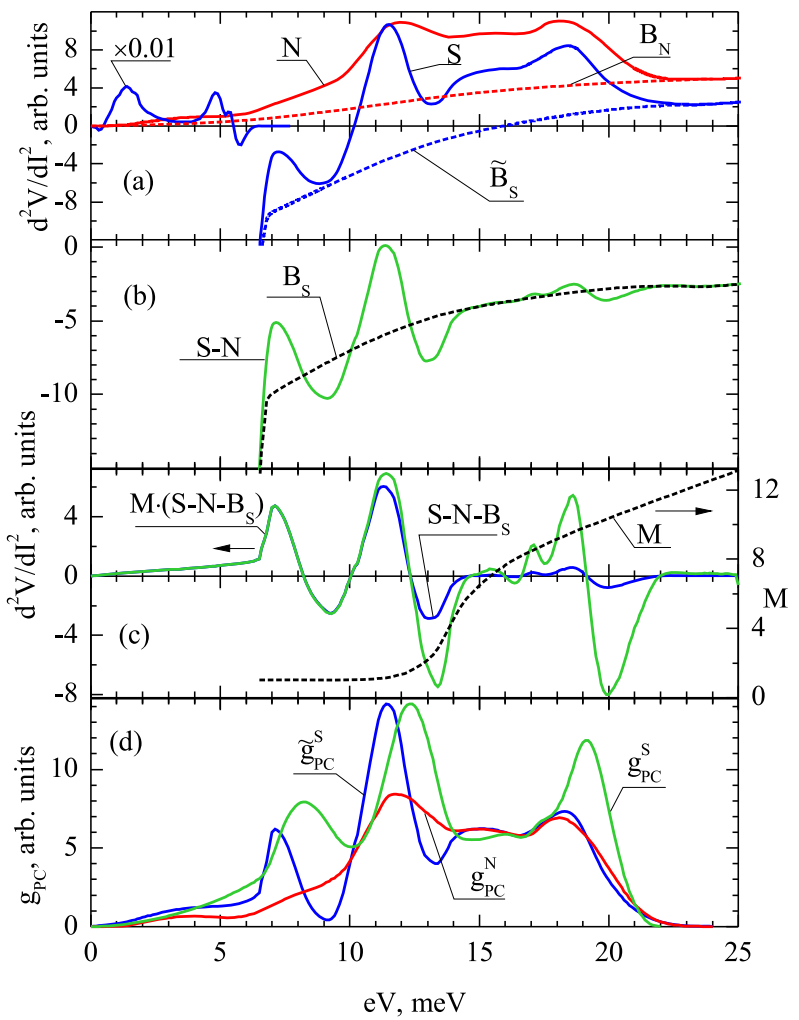

Figure 6. (a) EPI spectra of the Ta-Cu point contact in the normal and superconducting states. $T=1.7 \mathrm{~K}, R_{0}=73 \mathrm{ohm}$; the initial section of the superconducting curve containing the gap and nonequilibrium effects is scaled down by a factor of $100, \tilde{B}_{S}$ and $B_{N}$ are the background curves for the superconducting and normal spectra, respectively. (b) The difference between the superconducting and normal spectra and the assumed shape of the background curve. (c) Difference curve (after background subtraction), scaling curve M, and the difference curve multiplied by the scaling curve. (d) Point contact EPI functions recovered from the spectra for normal and superconducting states and from the superconducting contribution to the spectrum by integration of the corrected difference curve (c). For the convenience of comparison, the curve $g_{p c}^{S}$ is aligned in amplitude with the curve $\tilde{g}_{p c}^{S}$. The scale is the same in all panels of the Figure.

phonon mode by only an edge. The shape of this mode in the superconducting state basically differs from that in the normal state and is manifested as a peak rather than a shoulder; the phonon peak at about $11.3 \mathrm{mV}$ is also markedly sharper than that in the case of the $209 \mathrm{ohm}$ contact. If one refers to Fig.6(d), in the lowenergy part of the spectrum, attention is attracted by a much more pronounced increase in the peak amplitude upon transition to the superconducting state in comparison with the previous point contact; this supports the above idea 


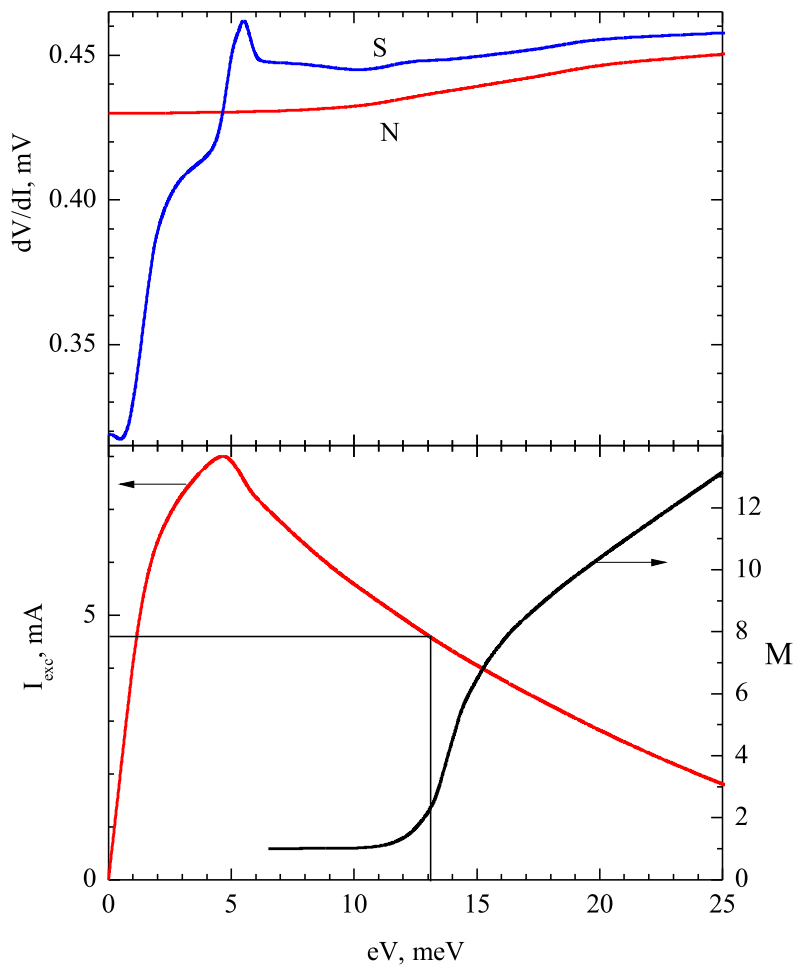

Figure 7. The first derivatives of the CVCs for a point contact whose characteristics are shown in Fig.6 in the normal $(\mathrm{N})$ and superconducting (S) states and excess current vs. the bias. The excess current panel shows the scaling curve $\mathrm{M}$ (see Fig.6) with designated excess current and voltage values corresponding to the start of the ascending part of the scaling curve.

that the contribution of the peripheral areas to the whole superconducting contribution increases with increasing point contact diameter. However, the superconducting contribution for the high-energy part of the spectrum is markedly lower in this case than for the previous contact. According to Fig.7, the suppression of the excess current with increasing contact bias is much greater in this case than in the former case, which is responsible for the observed result. Owing to these factors together, the scaling curve for correction of the high-frequency part of the superconducting contribution is approximately twice greater for this contact than that for the contact considered above.

Figure 8 shows the EPI spectra for the Ta-Cu point contact (one of the lowest-resistance point contacts involved in the study). When its resistance is $16 \mathrm{ohm}$, the diameter is close to $8.5 \mathrm{~nm}$, which is almost 4 times greater than that of the previous point contact (the volume is almost 60 times greater). Here the differences from the first two point contacts are much more pro-

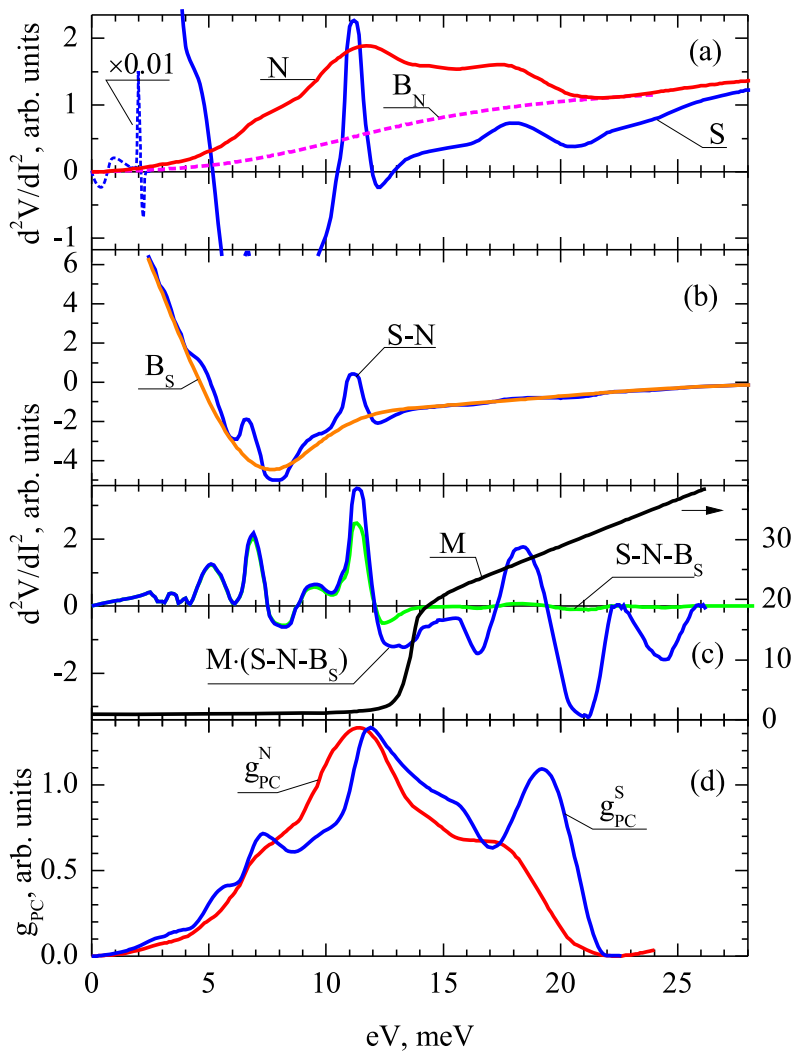

Figure 8. (a) EPI spectra of the Ta-Cu point contact in the normal and superconducting states; N: $T=4.6 K$; : $T=1.7 \mathrm{~K}, R_{0}=16 \mathrm{ohm}$, the initial dashed segment of the superconducting curve containing the gap and the nonequilibrium effects is scaled down by a factor of $100, B_{N}$ is the background curve for the normal spectrum. (b) The difference between the superconducting and normal spectra and the assumed shape of the background curve. (c) Difference curve (after background subtraction), scaling curve $\mathrm{M}$, and the difference curve multiplied by the scaling curve. (d) Point contact EPI functions recovered from the spectra in the normal state and from the superconducting contribution to the spectrum by integrating the corrected difference curve (c). For the convenience of comparison, the curve $g_{p c}^{S}$ is aligned in amplitude with the curve $g_{p c}^{N}$. The scale is the same in all panels of the Figure.

nounced. For this contact, the nonequilibrium effect is located almost immediately after the gap effect at a voltage slightly above $2 \mathrm{mV}$. This opens up the possibility to follow the fine structure of the initial section of the phonon spectrum of tantalum upon the transition to the superconducting state, because in the normal state, it is possible to follow only a gradual ascent of the spectrum in this energy range.

Figure 9 depicts the relevant EPI spectra in the su- 


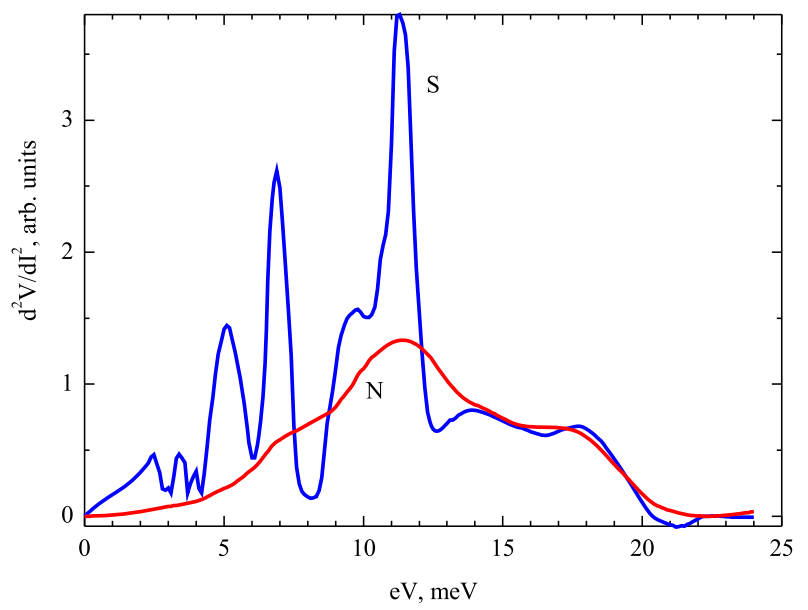

Figure 9. Second derivatives of the CVCs of the $16 \mathrm{ohm}$ Ta$\mathrm{Cu}$ point contact (see Fig.8) after background subtraction in the normal $(\mathrm{N})$ and superconducting $(\mathrm{S})$ states. The scale of the curves is the same as that in Fig. 8.

perconducting and normal states after background subtraction. For this contact in the superconducting state, the peak sharpening in the low-frequency region is much more pronounced than in the previous cases. The spectral amplitude also greatly increases; meanwhile these changes in the high-energy part of the spectrum are much weaker. In the difference curve [Fig.8(c)], the high-energy part is barely seen against the initial part before the correction.

Figure 10 shows the plots for differential resistances of this contact in the normal and superconducting states, excess current as a function of voltage, and the correction scaling curve. As follows from the Figure, the scaling curve amplitude is approximately 6 times higher than that of the previous contact, which is evidently attributable to two factors mentioned above: a pronounced increase in the amplitude of the low-energy part of the superconducting contribution, and a more pronounced suppression of the highenergy part caused by decreasing excess current.

\section{TA-TA HOMOCONTACTS}

The trends observed for heterocontacts are generally reproduced for homocontacts, although differences are also present: the spectra of homocontacts usually exhibit two nonequilibrium effects associated with attainment of the critical density of nonequilibrium quasi-particles in each bank. Figure 11 presents the EPI spectra of the

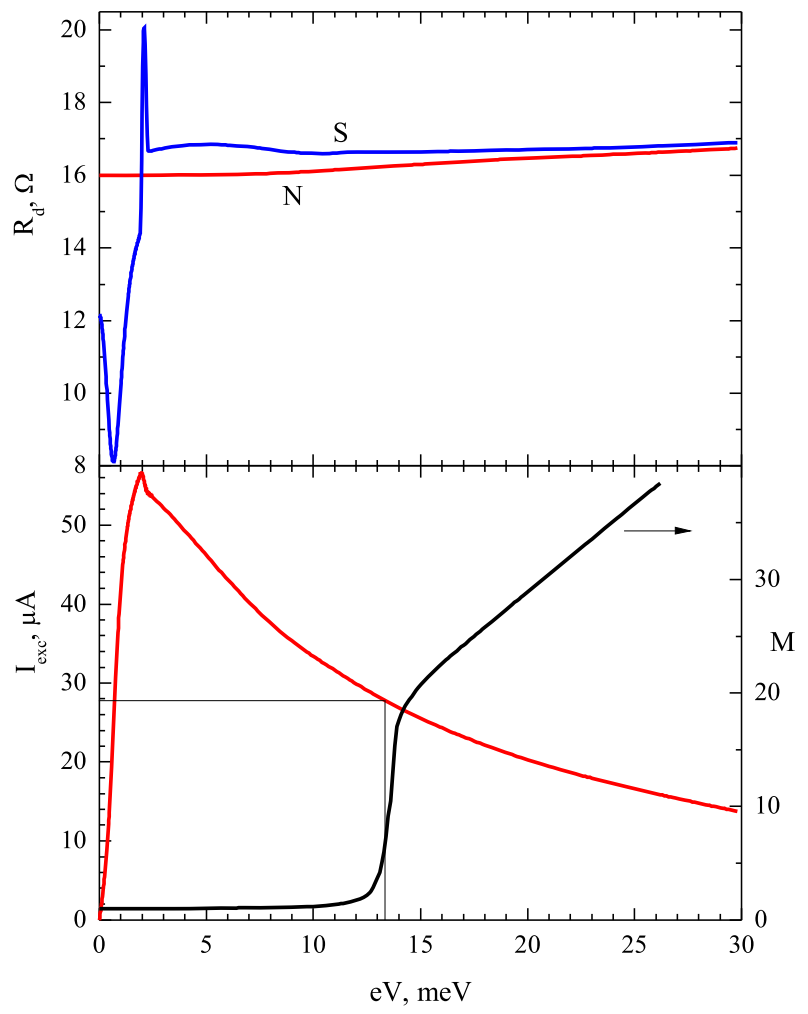

Figure 10. Differential resistance of the $\mathrm{Ta}-\mathrm{Cu}$ point contact whose characteristics are shown in Fig.8 in the normal $(\mathrm{N})$ and superconducting (S) states and excess current vs. the bias. The excess current panel shows the scaling curve $M$ (see Fig.8) with designated excess current and voltage values corresponding to the start of the ascending part of the scaling curve.

$64 \mathrm{ohm}$ Ta homocontact in the normal and superconducting states. Since the temperature during the measurements in the superconducting state proved to be somewhat higher for this contact than for heterocontacts, and because of the presence of two nonequilibrium effects, all low-energy region down to less than $10 \mathrm{meV}$ energies was inaccessible for observation of the behavior of the phonon effects. Therefore, the initial section of the EPI function recovered from the superconducting contribution to the spectrum was approximated by a parabola.

Figure 12 shows the differential resistances of the point contact in the normal and superconducting states, the energy dependence of the excess current, and the scaling curve. As can be seen in Fig.11(d), the sharpening and the intensity growth of the first peak upon the superconducting transition are markedly less pronounced in this case than for heterocontacts with a comparable resistance. Regarding the superconducting contribution to the spectrum [Fig.11(b)], its high-energy part is com- 


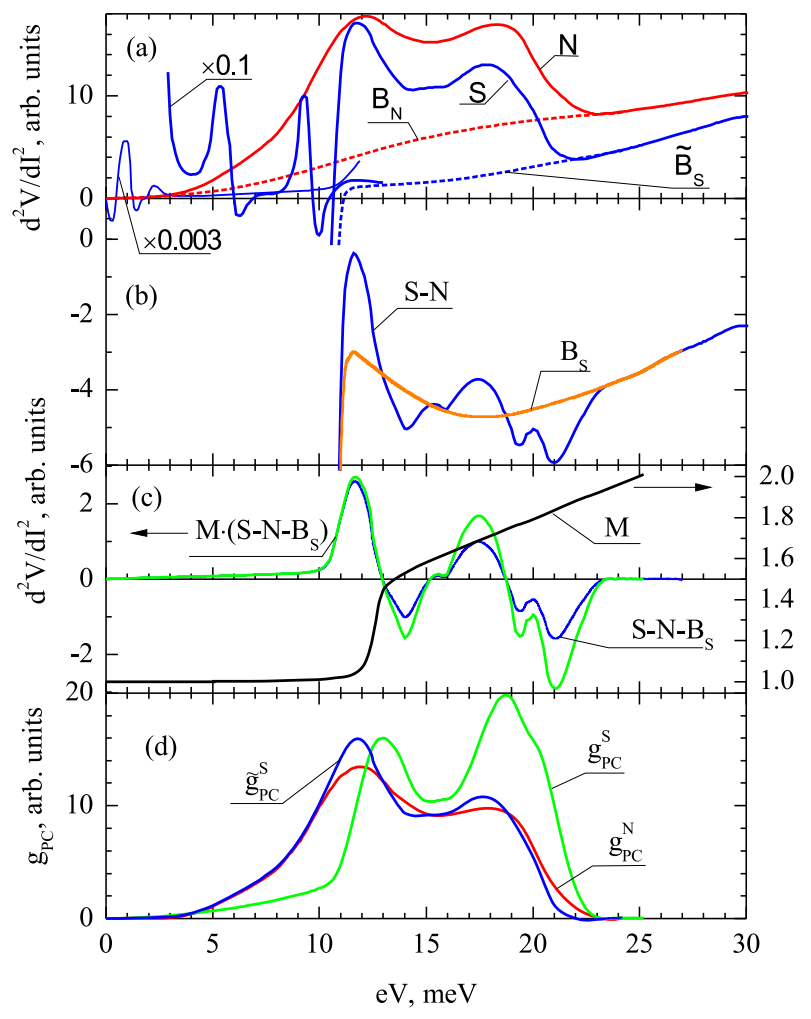

Figure 11. (a) EPI spectra of the Ta-Ta point contact in the normal and superconducting states. $\mathrm{N}: T=4.6 \mathrm{~K}$; $\mathrm{S}$ : $T=2.0 \mathrm{~K}, R_{0}=64 \mathrm{ohm}$, the initial segments of the superconducting curve containing the gap and nonequilibrium effects are scaled down by factors of 300 and 10 , respectively; $\tilde{B}_{S}$ and $B_{N}$ are the background curves for the normal and superconducting spectra, respectively. (b) The difference between the superconducting and normal spectra and the assumed shape of the background curve. (c) Difference curve (after background subtraction), scaling curve $\mathrm{M}$, and the difference curve multiplied by the scaling curve. (d) Point contact EPI functions recovered from the spectra for normal and superconducting states and from the superconducting contribution to the spectrum by integration of the corrected difference curve (c). The scale is the same in all panels of the Figure.

parable in intensity to the low-energy one. Therefore, the correction scaling curve $\mathrm{M}$ is relatively small in amplitude as compared with that of the heterocontacts.

Characteristics for the markedly lower-resistance homocontact (17 ohm) are shown in Figs. 13 and 14. For this contact, the nonequilibrium effect was found to occur at less than $5 \mathrm{mV}$ energy; therefore, the transformation of the soft mode during the superconducting transition of the contact was accessible for observation. Like for heterocontacts, it is converted into a peak. As follows from Fig.13(d), pronounced sharpening and an ampli-

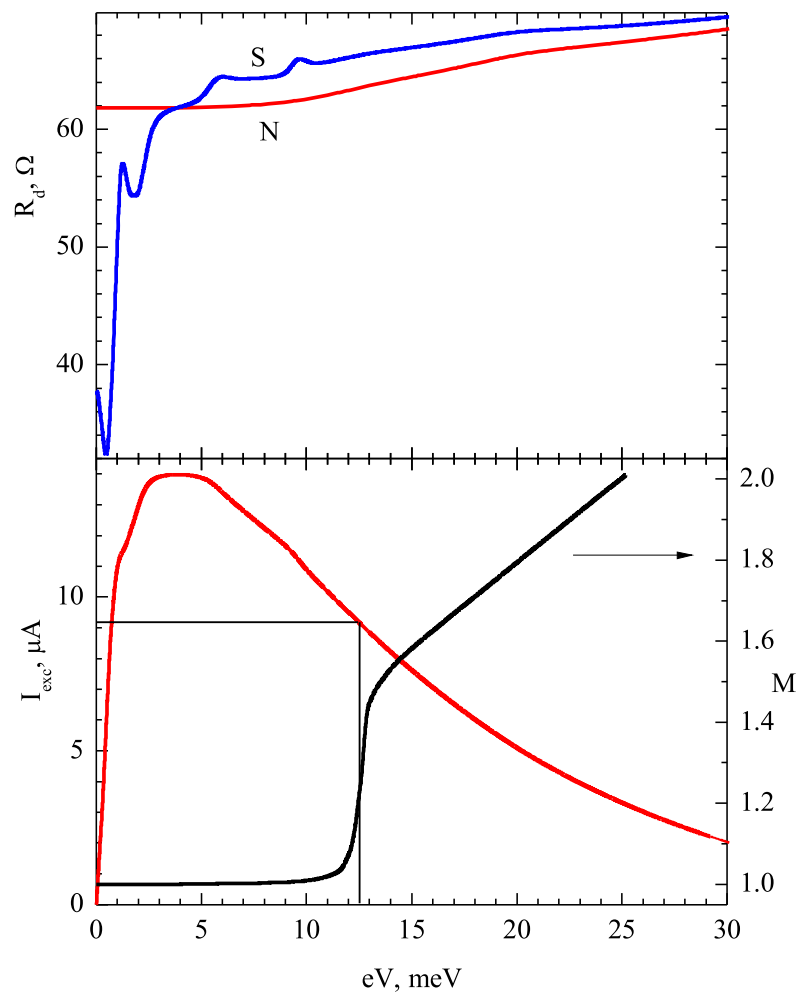

Figure 12. Differential resistances of the Ta-Ta point contact whose characteristics are shown in Fig.11 in the normal $(\mathrm{N})$ and superconducting (S) states and excess current vs. the bias. The excess current panel shows the scaling curve $M$ (see Fig.11) with designated excess current and voltage values corresponding to the start of the ascending part of the scaling curve.

tude increase of the first peak are also observed for this contact. Regarding the high-energy part of the spectrum, the changes induced by the superconducting transition are moderate. Nevertheless, a comparison with the proportion of the high-energy part of the superconducting contribution for the heterocontact with virtually the same resistance [Fig.8(c)] demonstrates that this proportion is quite comparable in intensity with that of the low-energy part; therefore, the correction scaling curve is an order of magnitude smaller.

\section{RESULTS AND DISCUSSION}

The conducted study of the ballistic point contacts based on tantalum demonstrated that all deviations from the predictions of the theoretical model are caused by involving a region that is about the size of the coherence length, which is adjacent to the constriction, in the formation of the EPI spectra upon the transition of the con- 


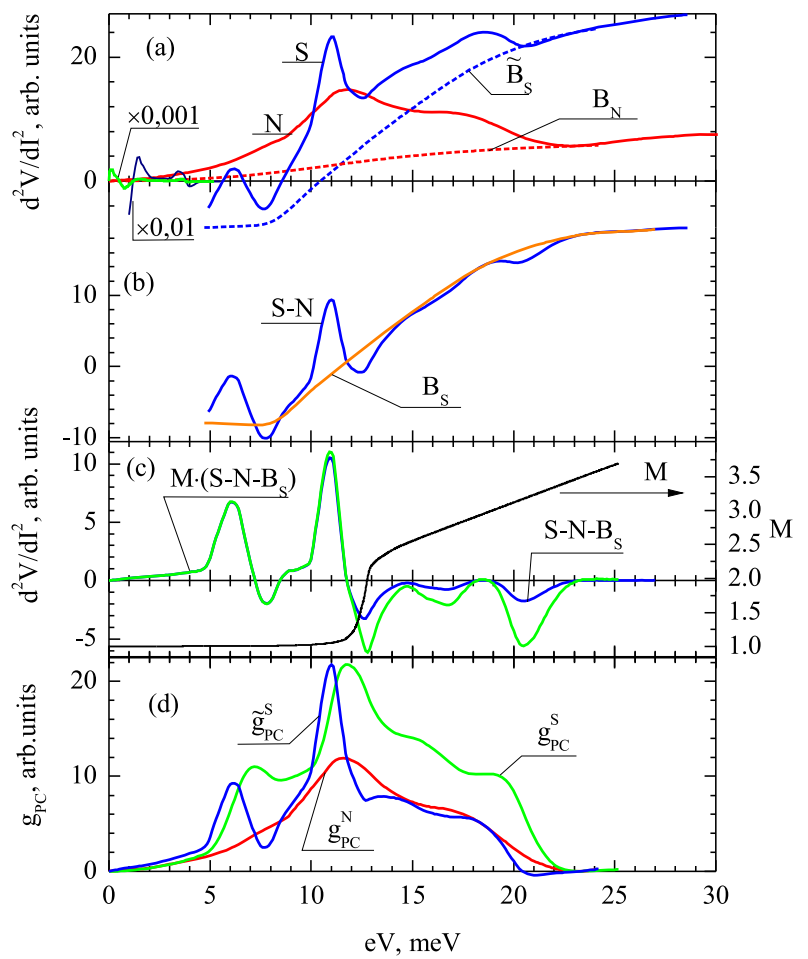

Figure 13. a) EPI spectra of the Ta-Ta point contact in the normal and superconducting states; $\mathrm{N}: T=4.6 K$; $T=2.55 K, R_{0}=17 \mathrm{ohm}$; the initial segments of the superconducting curve corresponding to the critical current and gap and nonequilibrium effects are scaled down by factors of 1000 and 100, respectively, $\tilde{B}_{S}$ and $B_{N}$ are the background curves for the normal and superconducting spectra. (b) The difference between the superconducting and normal spectra and the assumed shape of the background curve. (c) Difference curve (after background subtraction), scaling curve $\mathrm{M}$, and the difference curve multiplied by the scaling curve. (d) Point contact EPI functions recovered from the spectra for normal and superconducting states and from the superconducting contribution to the spectrum by integrating the corrected difference curve (c). For the convenience of comparison, the curve $g_{p c}^{S}$ is aligned in amplitude with the curve $\tilde{g}_{p}^{S} c$. The scale is the same in all panels of the Figure.

tact to the superconducting state. Apart from other differences, these contacts show a pronounced dependence of the superconducting contribution to the spectrum on the contact bias and on the resistance. This dependence, caused by partial suppression of the excess current by nonequilibrium quasi-particles, complicates the recovery of the EPI function from this contribution and requires a correction of its shape to ensure the fulfilment of the rule of sums, after subtraction of the superconducting background.

The search for the shape and intensity of the correction

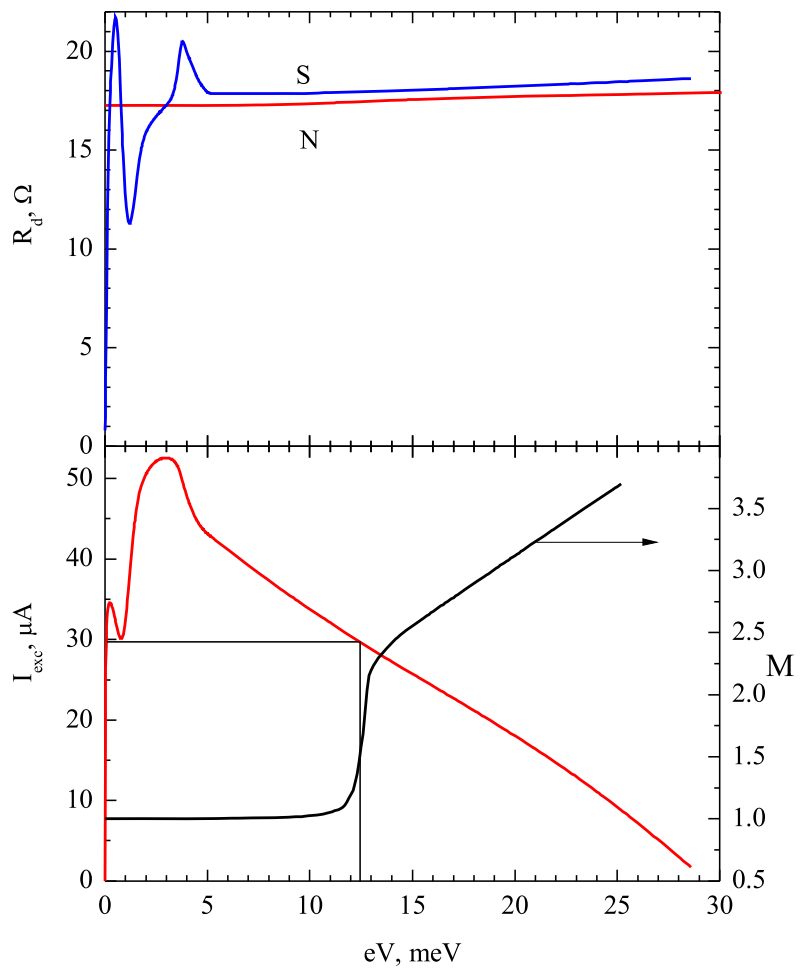

Figure 14. Differential resistance of the Ta-Ta point contact whose characteristics are shown in Fig.13 in the normal (N) and superconducting (S) states and excess current vs. the bias. The excess current panel shows the scaling curve $\mathrm{M}$ (see Fig.13) with designated excess current and voltage values corresponding to the start of the ascending part of the scaling curve.

scaling curve was performed separately for each of the considered point contacts. As has already been noted, the scaling curve intensity varies over wide limits, which is reflected in Fig.15(a). However, the shapes of these curves were similar. Figure 15(b) shows all scaling curves $\mathrm{M}$ reduced to the same amplitude. Moreover, the same correction curve proved to be applicable, with only the amplitude being varied. For example, the curves for contacts 4 and 5 have the same shape, but differ in the amplitude approximately 3.7 -fold. Attention is attracted by the step present in all correction curves at similar energies with approximately the same amplitude on a reduced scale. The curves for the excess current as a function of the bias show no peculiar effects at these energies, with the magnitude of the excess current smoothly descending. By analogy with appearance of a step along the dependence of the excess current on the bias, associated with the attainment of the critical concentration of nonequilibrium quasi particles above the gap in the near-contact region, one can assume that there exists some threshold 


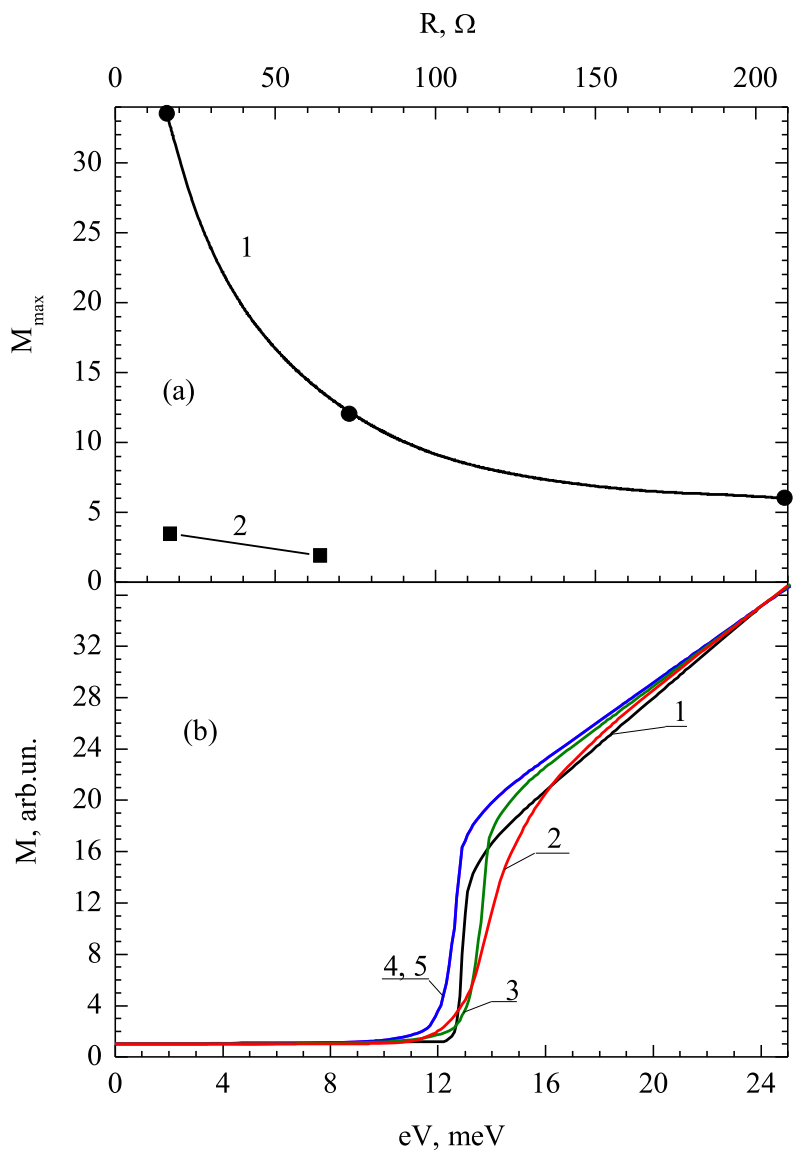

Figure 15. (a) A correction scaling curve at the phonon spectrum boundary at $23 \mathrm{meV}$ as a function of resistance of heteroand homocontacts. (b) Correction scaling curves for contacts with different resistances reduced to the same amplitude; (1) Ta-Cu, $R=209$ ohm; (2) Ta-Cu, $R=73$ ohm; (3) Ta-Cu, $R$ $=16$ ohm; (4) Ta-Ta, $R=64$ ohm; (5) Ta-Ta, $R=17$ ohm. Curves 4 and 5 have the same shape but differ in the amplitude approximately 3.7-fold (see Figs.12 and 14).

concentration of Andreev electrons determined by the excess current, below which the efficiency of reabsorption of nonequilibrium phonons sharply decreases, resulting in a sharp decrease in the superconducting contribution to the spectrum. Further decreases in the concentration of Andreev electrons with increasing contact bias lead to a smooth decline of the superconducting contribution to the spectrum, which is reflected in the shape of the correction scaling curves. This assumption is supported by the comparison of homo- and heterocontacts. When the resistances are similar, the amplitude of the correction curves is much smaller for homocontacts, which is due to the doubled excess current. However, as can be seen in Fig.15, there are no obvious differences between the shapes of the correction curves for homo- and heterocontacts.

\section{BRIEF CONCLUSIONS}

1. The tantalum-based ballistic point contacts in the superconducting state show deviations from the predictions of theoretical models due to the involvement of a near-contact region with a size that is about equal to the reduced coherence length $\zeta$, in the formation of EPI spectra.

2. The relative value of the superconducting contribution to the EPI spectrum depends on the contact resistance and increases with increasing contact diameter.

3. The superconducting contribution decreases with increasing contact bias due to the suppression of excess current by the nonequilibrium quasi-particles, which leads to violation of the formulated rule of sums.

4. Although the EPI functions are proportional to the first derivative of the excess current, the presence of the superconducting background and the need to correct the amplitude of the superconducting spectral contribution caused by violation of the rule of sums, bring about the necessity to recover the EPI functions from the CVC second derivatives.

5. The procedure of recovering the EPI spectral function described in detail can be used to analyze the characteristics of ballistic point contacts based on a broad range of superconductors.

\section{ACKNOWLEDGMENTS}

This study was supported by the National Academy of Sciences of Ukraine as a part of project FTs 4-19.

In conclusion, the author would like to express gratitude to A.V. Khotkevich for numerous tips, comments, and discussions. 
[1] I.K. Yanson and A. V. Khotkevich, Atlas Mikrokontaktnykh Spektrov Elektron- Fononnogo Vzaimodeistviya v Metallakh (Naukova Dumka, Kyiv, 1986) [A. V. Khotkevich and I. K. Yanson, Atlas of Point-Contact Spectra of Electron- Phonon Interaction in Metals, (Kluwer Academic Publishers, Boston, 1995)].

[2] Y. G. Naidyuk and I. K. Yanson, Point-Contact Spectroscopy (Springer, New York, 2005).

[3] I. K. Yanson, I. O. Kulik, A. N. Omel'yanchuk, R. I. Shekhter, and Y. V. Sharvin, Phenomenon of Charge Carrier Energy Redistribution in Metal Microcontacts at low Temperatures (Discoveries in the USSR, VNIIPI, Moscow, 1986), p. 18 (Diploma No. 328, Discoveries. Invent. (1987), No. 40, p. 3).

[4] I. O. Kulik, A. N. Omel'yanchuk, and R. I. Shekhter, Fiz. Nizk. Temp. 3, 1543 (1977) [Sov. J. Low Temp. Phys. 3, 740 (1977)].

[5] V. A. Khlus and A. N. Omel'yanchuk, Fiz. Nizk. Temp. 9, 373 (1983) [Sov. J. Low Temp. Phys. 9, 189 (1983)].

[6] V. A. Khlus, Fiz. Nizk. Temp. 9, 985 (1983) [Sov. J. Low Temp. Phys. 9, 510,(1983)].
[7] N. L. Bobrov, V. V. Fisun, O. E. Kvitnitskaya, V. N. Chenobai, and I. K. Yanson, Fiz. Nizk. Temp. 38, 480 (2012) [Low Temp. Phys. 38, 373 (2012)]; arXiv:1207.6486.

[8] N. L. Bobrov, A. V. Hotkevich, G. V. Kamarchuk, and P. N. Chubov, Fiz. Nizk.Temp. 40, 280 (2014) [Low Temp. Phys. 40, 215 (2014)]; arXiv:1405.6869.

[9] N. L. Bobrov, Fiz. Nizk. Temp. 41, 768 (2015) [Low Temp. Phys. 41, 595 (2015)]; arXiv:1512.04082.

[10] I. K. Yanson, V. V. Fisun, N. L. Bobrov, and L. F. Rybal'chenko, JETP Letters 45, 425 (1987) [JETP Lett. 45, 543 (1987)]; arXiv:1602.04356.

[11] I. K. Yanson, N. L. Bobrov, L. F. Rybal'chenko, and V. V. Fisun, Fiz. Nizk. Temp. 13, 1123 (1987) [Sov. J. Low Temp. Phys. 13, 635 (1987)]; arXiv:1512.03917.

[12] I. K. Yanson, L. F. Rybal'chenko, N. L. Bobrov, and V. V. Fisun, Fiz. Nizk. Temp. 12, 552 (1986) [Sov. J. Low Temp. Phys. 12, 313 (1986)]; arXiv:1512.00684.

[13] N. L. Bobrov, L. F. Rybal'chenko, V. V. Fisun, and I. K. Yanson, Fiz. Nizk. Temp. 13, 611 (1987) [Sov. J. Low Temp. Phys. 13, 344 (1987)]; arXiv:1512.01800. 